\title{
3D AND 1D SIMULATIONS OF DIESEL PARTICULATE FILTER (DPF)
}

\author{
NGUYEN MINH PHU, LE THANH DANH \\ Faculty of Mechanical Engineering, Industrial University of Ho Chi Minh City \\ nmphu2016@gmail.com
}

\begin{abstract}
DPF is an important device in the exhaust system of Diesel engine. In this paper we simulate velocity and pressure distributions in DPF to determine kinematic and hydraulic characteristics. This will provide the basis for designing and selecting size of channels in DPF. Numerical simulations were made using ANSYS Fluent commercial software and OpenFOAM open-source software. The results show that the difference between the two softwares is negligible. A compact 1D mathematical model developed based on the Darcy equation, momentum equation and continuity equation. The mathematical model solved by shooting method for boundary value problem. Simulation results from 1D and 3D approaches are very coincident.
\end{abstract}

Keywords. Diesel particulate filter, Numerical simulation, Porosity model, OpenFOAM, Fluent.

\section{INTRODUCTION}

The OpenFOAM (Open Field Operation and Manipulation) CFD Toolbox is a free, open source CFD software package produced by OpenCFD Ltd. It has a large user base across most areas of engineering and science, from both commercial and academic organizations. OpenFOAM has an extensive range of features to solve anything from complex fluid flows involving chemical reactions, turbulence and heat transfer, to solid dynamics and electromagnetics. It includes tools for meshing, notably snappyHexMesh, a parallelized mesher for complex CAD geometries, and for pre- and post-processing. Almost everything (including meshing, and pre- and post-processing) runs in parallel as standard, enabling users to take full advantage of computer hardware at their disposal.

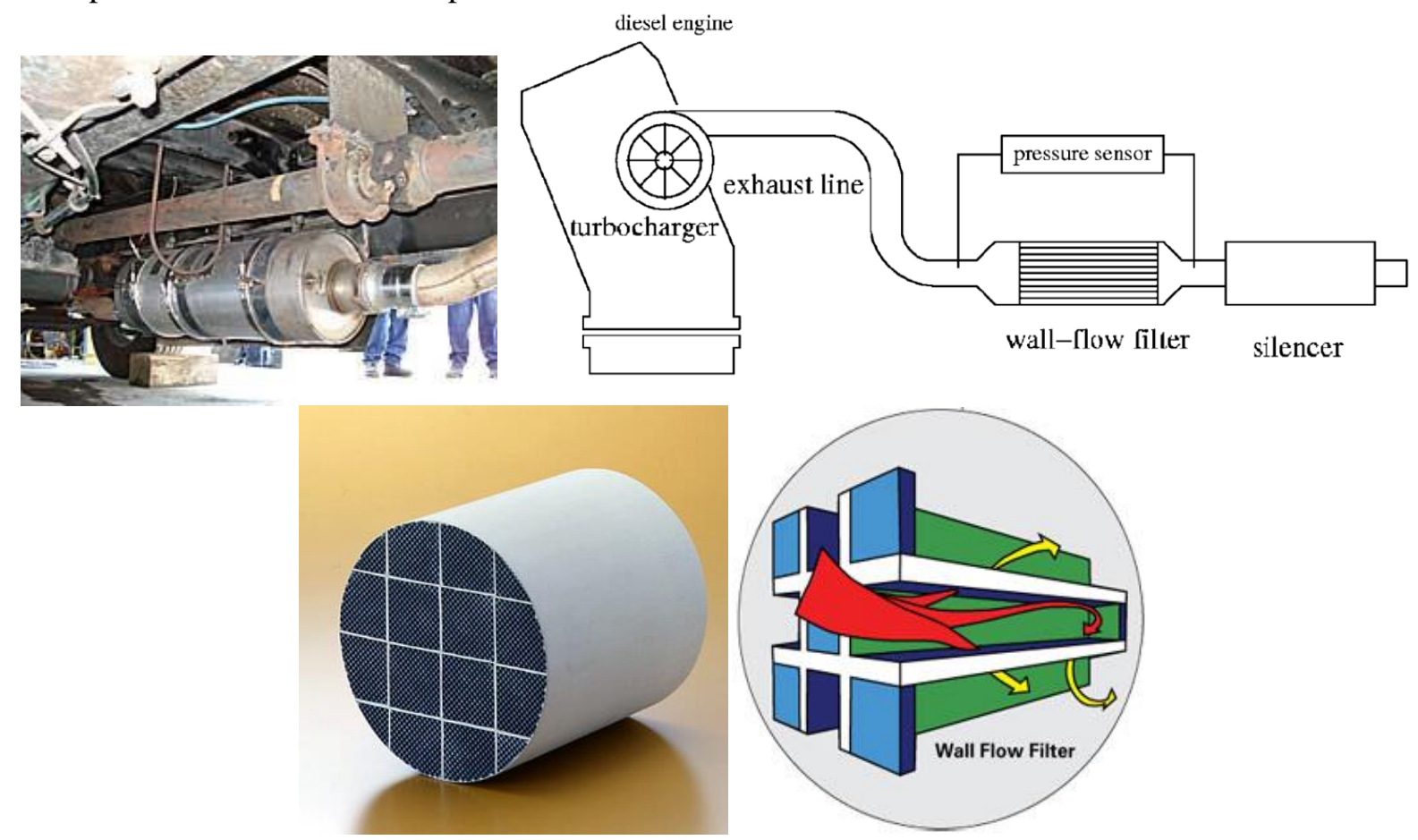

Figure 1: Diesel particulate filter.

ANSYS FLUENT software contains the broad physical modeling capabilities needed to model flow, turbulence, heat transfer, and reactions for industrial applications ranging from air flow over an aircraft wing to combustion in a furnace, from bubble columns to oil platforms, from blood flow to semiconductor manufacturing, and from clean room design to wastewater treatment plants. Special models that give the 
software the ability to model in-cylinder combustion, aeroacoustics, turbomachinery, and multiphase systems have served to broaden its reach.

During the last few years, an increased demand of diesel engine is caused by the customers because of its improved performance and low fuel consumption. However, the diesel engine still has some disadvantages, two of which are its high $\mathrm{NO}_{\mathrm{x}}$ and particulate matter (PM) emissions. PM emissions can be reduced by internal purification technology such as fuel combustion optimization. Nevertheless, as the emission standards become more and more stringent, the after-treatment devices become the indispensable tools. Among the after-treatment devices, a diesel particulate filter (DPF) placed in the exhaust line of vehicle is a well-established tool for PM emissions reduction.

At present, the dominant DPF configuration is that of a wall-flow honeycomb multi-channel structure with alternatively blocked inlet and outlet channels, whose structure is shown in Figure 1. Thus the exhaust gas is forced through the porous walls and the particles are captured inside the porous walls or on the surface of the inlet channels. The deposited particles increase the pressure loss of DPFs, and excessive pressure loss penalizes the engine fuel economy. Accordingly, the particles must be oxidized to avoid the impact of pressure loss on the fuel economy, which is called regeneration process. In this study we simulate velocity and pressure distributions in DPF to determine kinematic and hydraulic characteristics. This will provide the basis for designing and selecting size of channels such as channel width, channel length, wall thickness, flow rate in DPF. Numerical simulations were made using the above-mentioned ANSYS Fluent and OpenFOAM softwares.

\section{3D SIMULATION BY USING OPENFOAM AND FLUENT SOFTWARES}

Porous media in diesel particulate filter (DPF) is simulated using OpenFOAM 1.7.1. The obtained result is compared with that of Fluent software. A 3D computational domain is extracted from a DPF. Input parameters are get from previous study such as size of DPF, properties of porous, boundary conditions, etc. The rhoPorousSimpleFoam is used as a solver in OpenFOAM. Compressible and laminar flow is considered in the simulation. Energy equation is also included in model. The 3D domain can be seen in Figures 2 and 3.

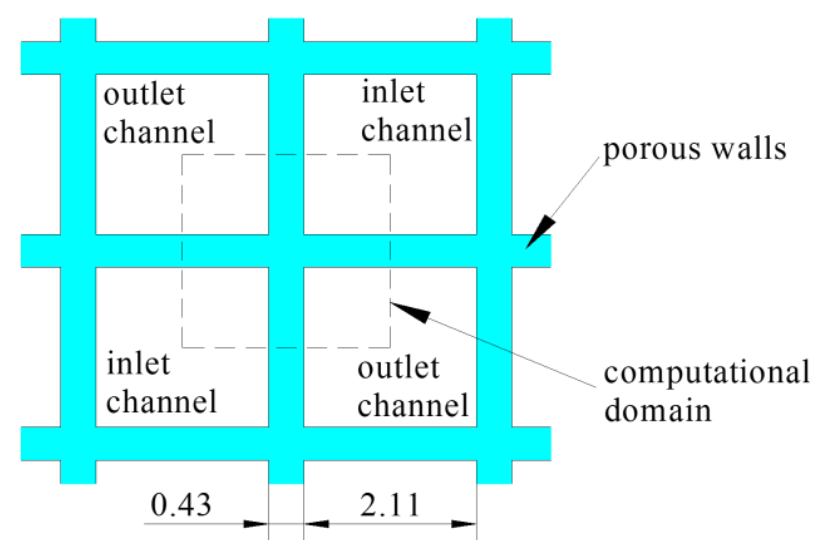

Figure 2: Cross section of DPF channels.

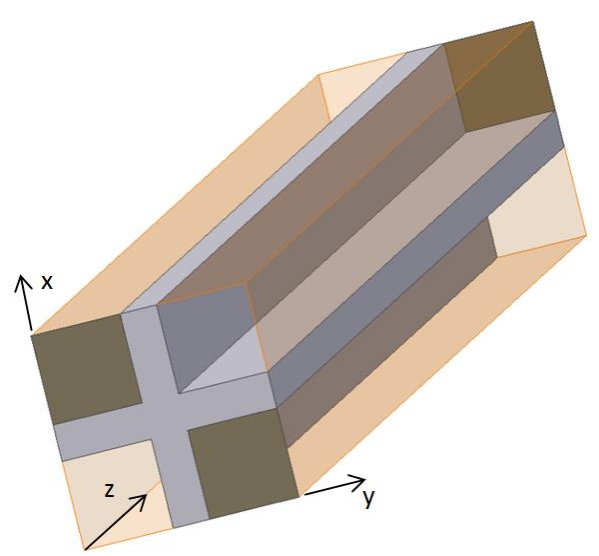

Figure 3: 3D Computational domain. 
The problem can be depicted briefly as follows:

- Features of the problem

- Compressible and laminar flow

- Included energy equation

- Porous:

- Viscous resistance: $1 \mathrm{e} 12 \mathrm{~m}^{-2}$

- Inertial resistance: $5 \mathrm{e} 8 \mathrm{~m}^{-1}$

- B.C.:

○ Mass flow rate inlet: $3 \mathrm{e}-5 \mathrm{~kg} \cdot \mathrm{s}^{-1}$

○ Temperature inlet: $652 \mathrm{~K}$

- Pressure outlet: $101325 \mathrm{~Pa}$

- Properties:

- Dynamic viscosity is based on Sutherland's law with two coefficients as $\mathrm{C}_{1}=1.4792 \mathrm{e}-6 \mathrm{~kg} / \mathrm{m} \cdot \mathrm{s} \cdot \mathrm{K}^{1 / 2} ; \mathrm{C}_{2}=116 \mathrm{~K}$

$\circ \quad c_{\mathrm{p}}=1007 \mathrm{~J} \cdot \mathrm{kg}^{-1} \cdot \mathrm{K}^{-1}$

○ Molecular weight: 28.9 g.mol ${ }^{-1}$

- Ideal gas

- Geometry:

- Channel width: $2.11 \mathrm{~mm}$

- Channel length: $304.8 \mathrm{~mm}$

○ Wall thickness: $0.432 \mathrm{~mm}$

Results at $200^{\text {th }}$ iteration are presented in this section. The same problem is also carried out using Fluent in order to show comparisons. Figure 4 presents residuals from 2 tools.

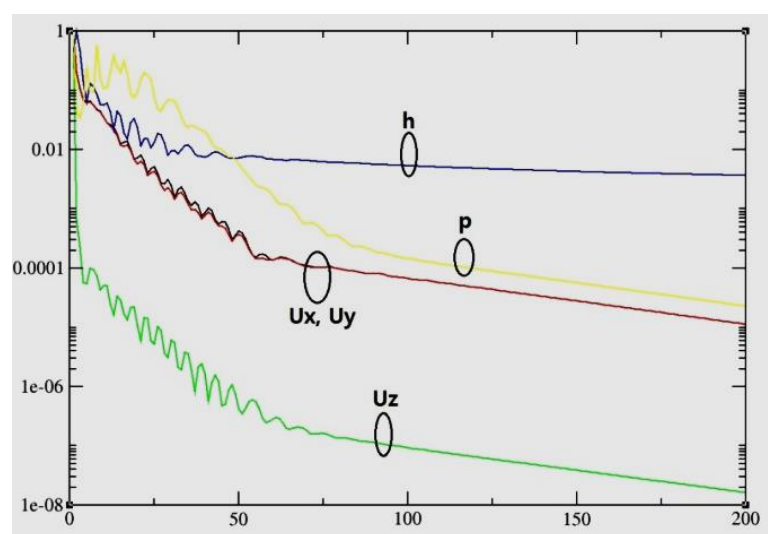

a) OpenFOAM

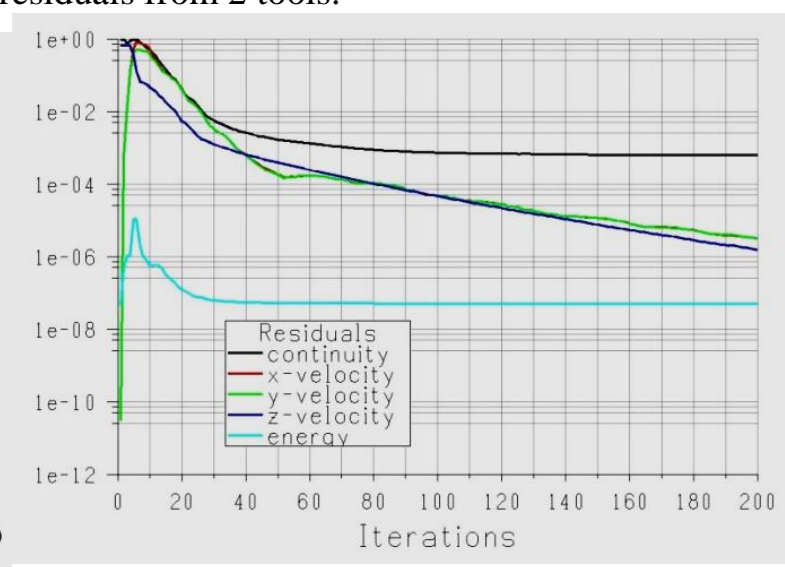

b) Fluent

Figure 4: Residuals.

Figures 5 and 6 show pressure and velocity magnitude contour from 2 CFD tools. We can see that pressure drop of the gas across the filter is about $1640 \mathrm{~Pa}$. 


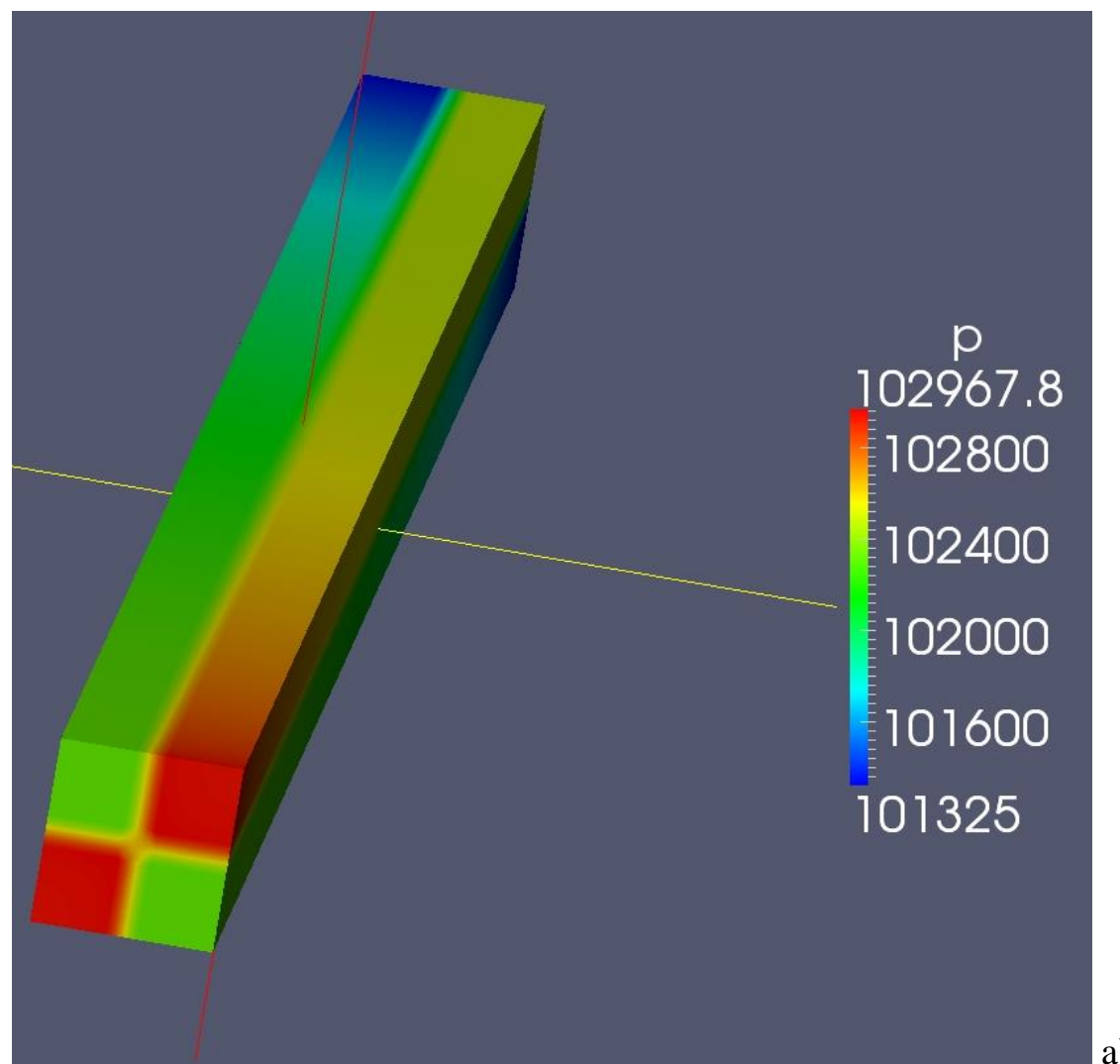

a)

$1.03 e+05$

$1.03 e+05$

$1.03 e+05$

$1.03 e+\square 5$

$1.03 e+05$

$1.03 e+05$

$1.03 e+05$

$1.02 e+05$

$1.02 e+05$

$1.02 e+05$

$1.02 e+05$

$1.02 e+05$

$1.02 e+05$

$1.02 e+05$

$1.02 e+05$

$1.02 e+\square 5$

$1.02 e+05$

$1.02 e+\square 5$

$1.02 \mathrm{e}+05$

$1.01 e+05$

$1.01 e+05$

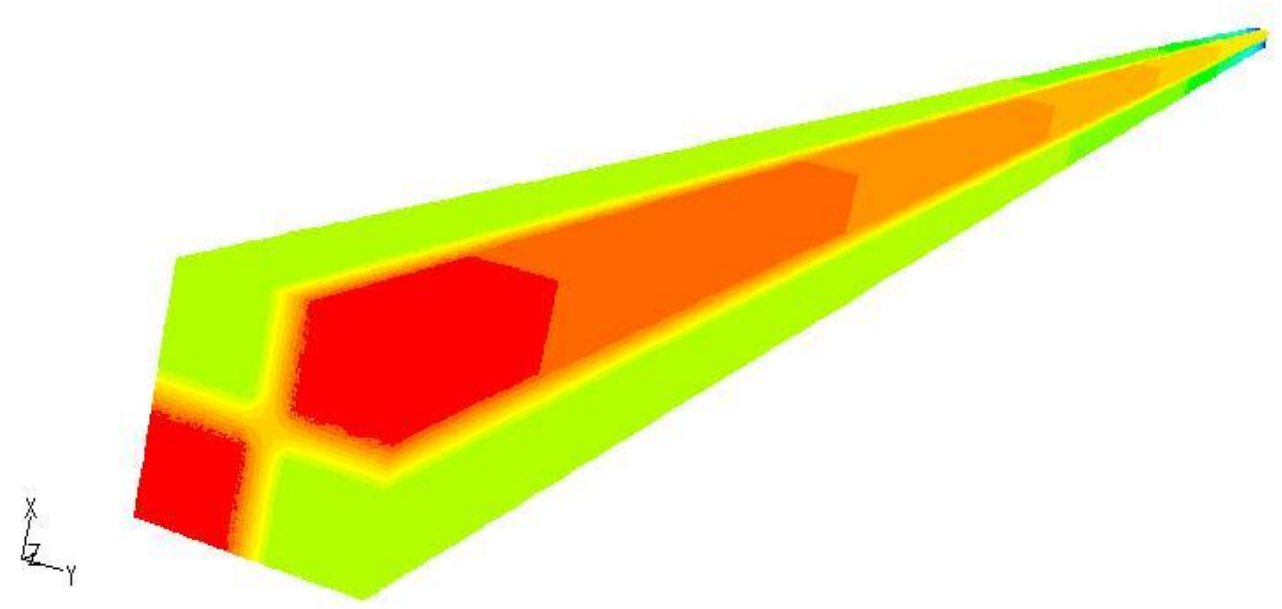

b)

Figure 5: Absolute pressure. a) OpenFOAM, b) Fluent. 

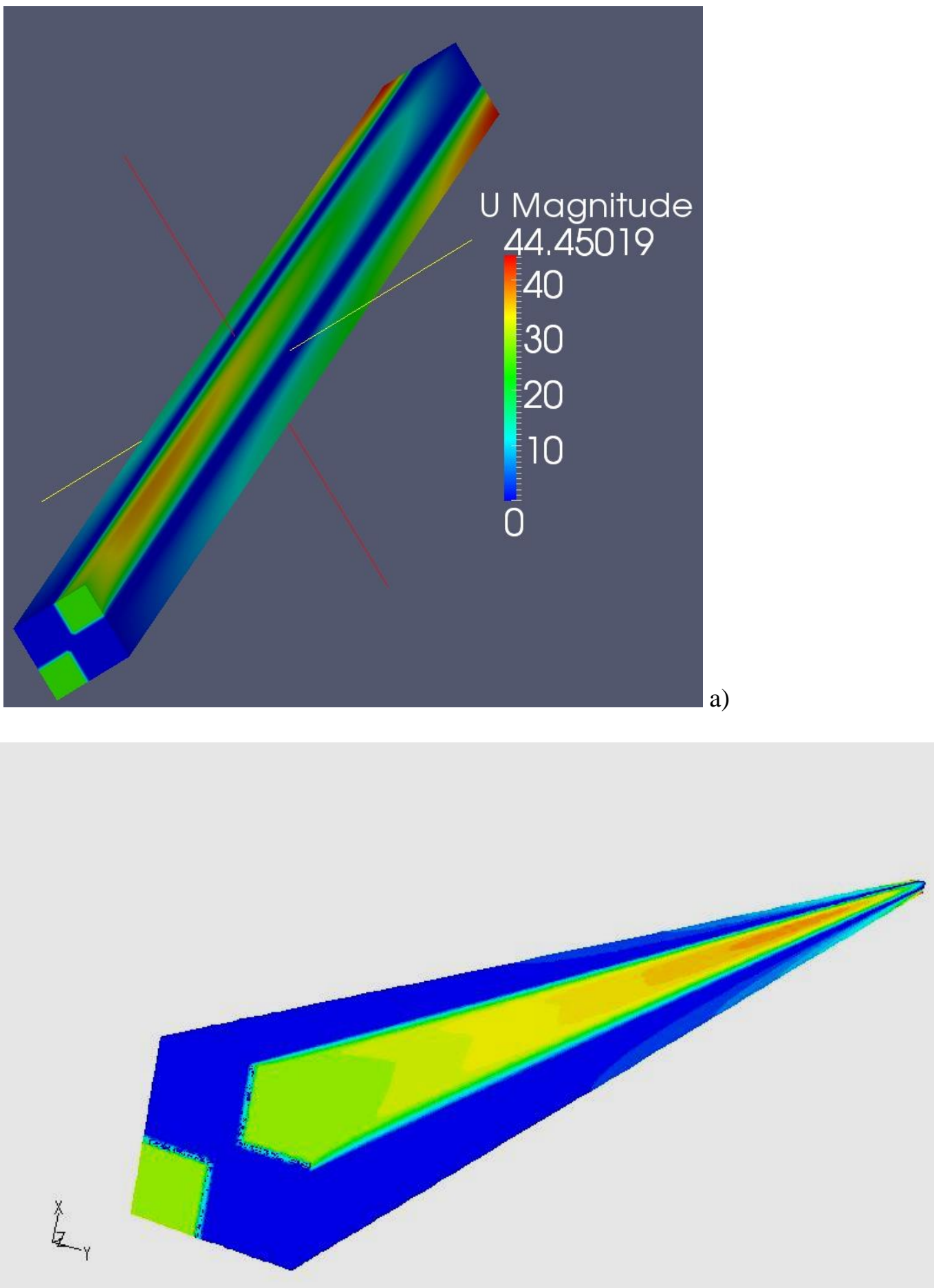

b)

Figure 6: Velocity magnitude. a) OpenFOAM, b) Fluent.

Figure 7 presents velocity vector at typical cross section a long z-direction. It should be noted that magnitude of the vector represents $\mathrm{x}$ and $\mathrm{y}$ velocity. Color of vector represents magnitude of velocity. 


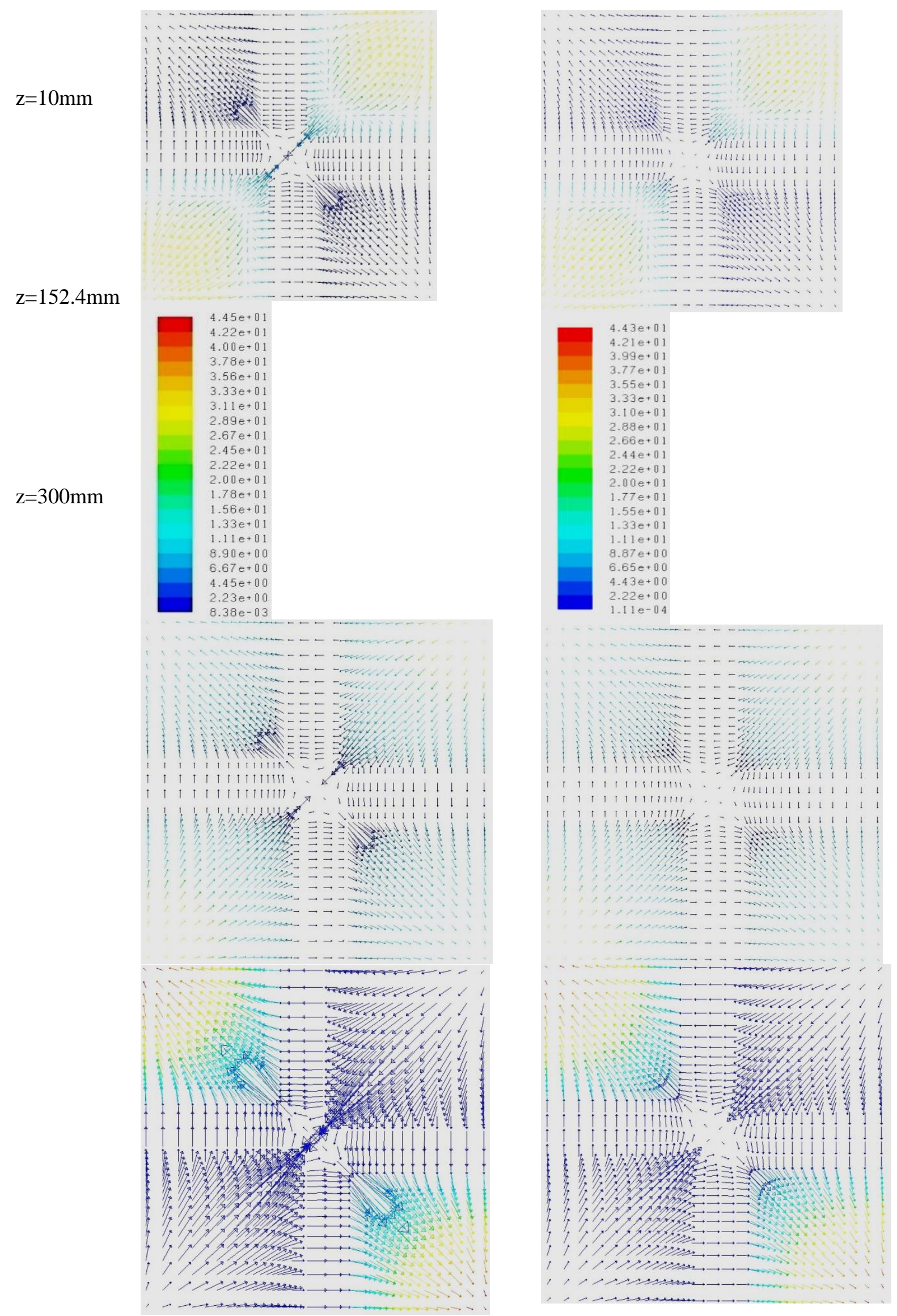

Figure 7: Velocity vector at cross sections. 
Figure 8 shows lines where data is used to display in Figures 9-10.

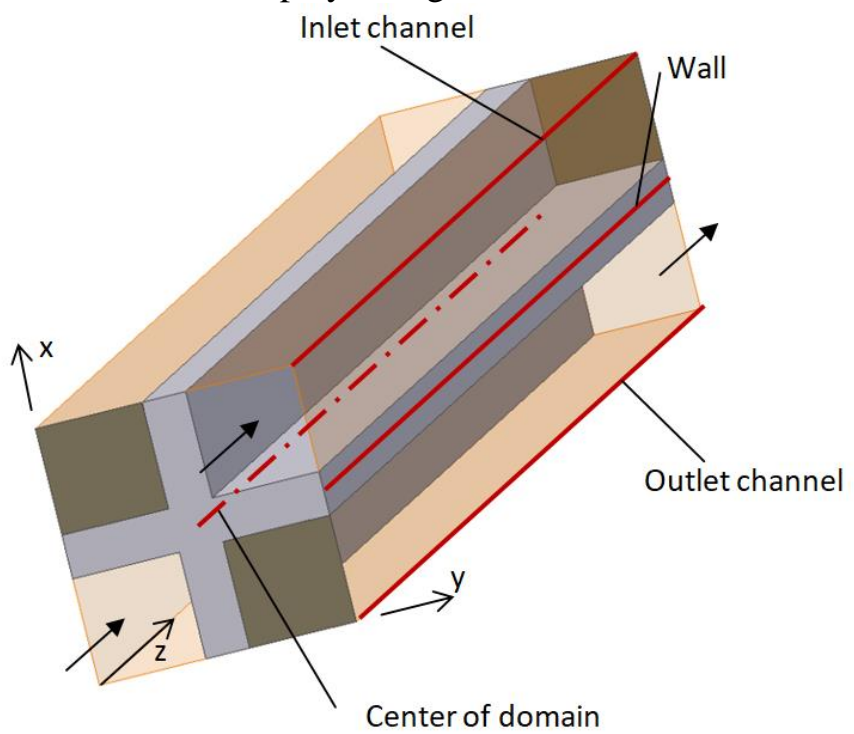

Figure 8: Lines for displaying data.

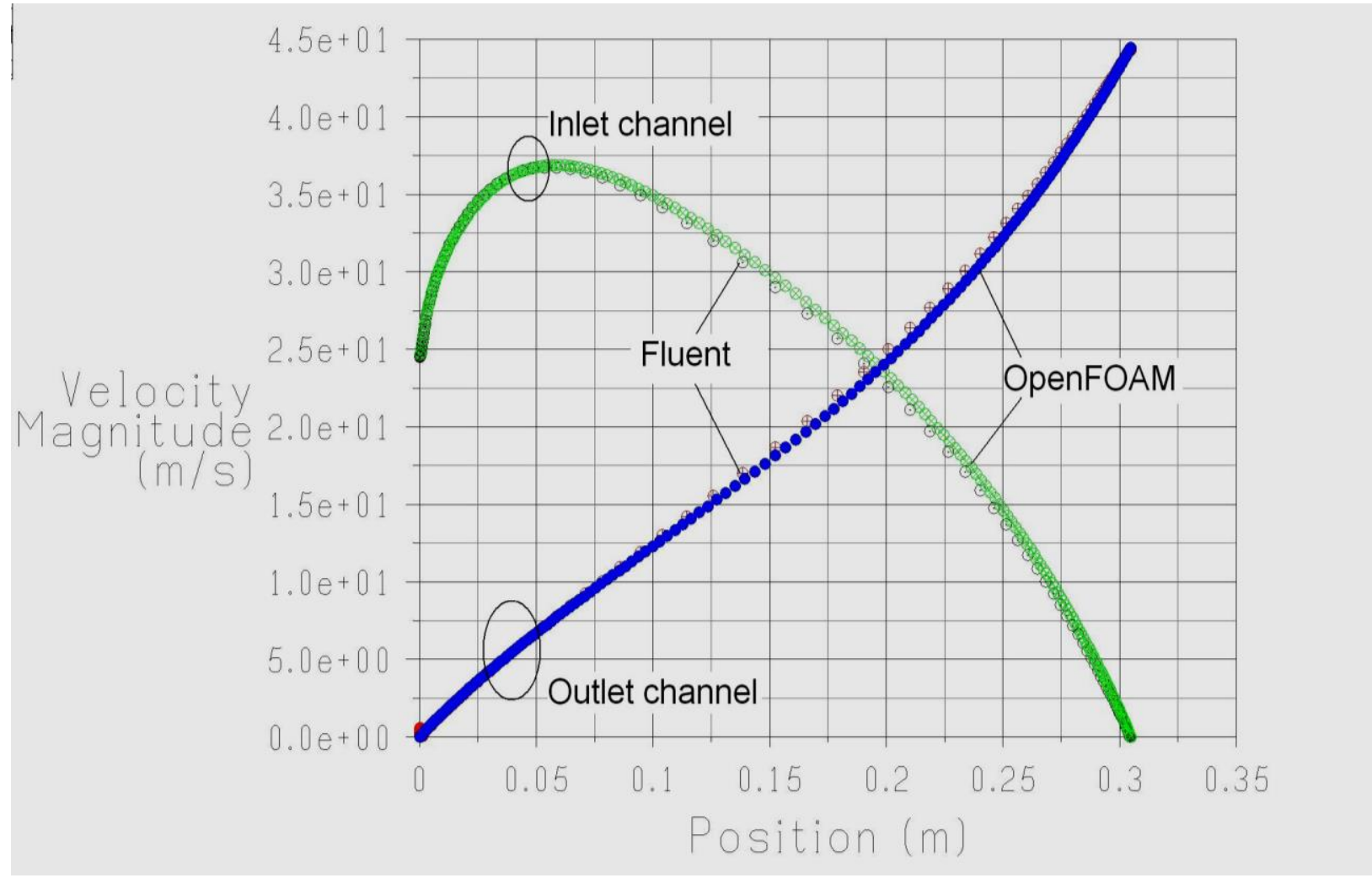

Figure 9: Velocity profiles along z-direction. 


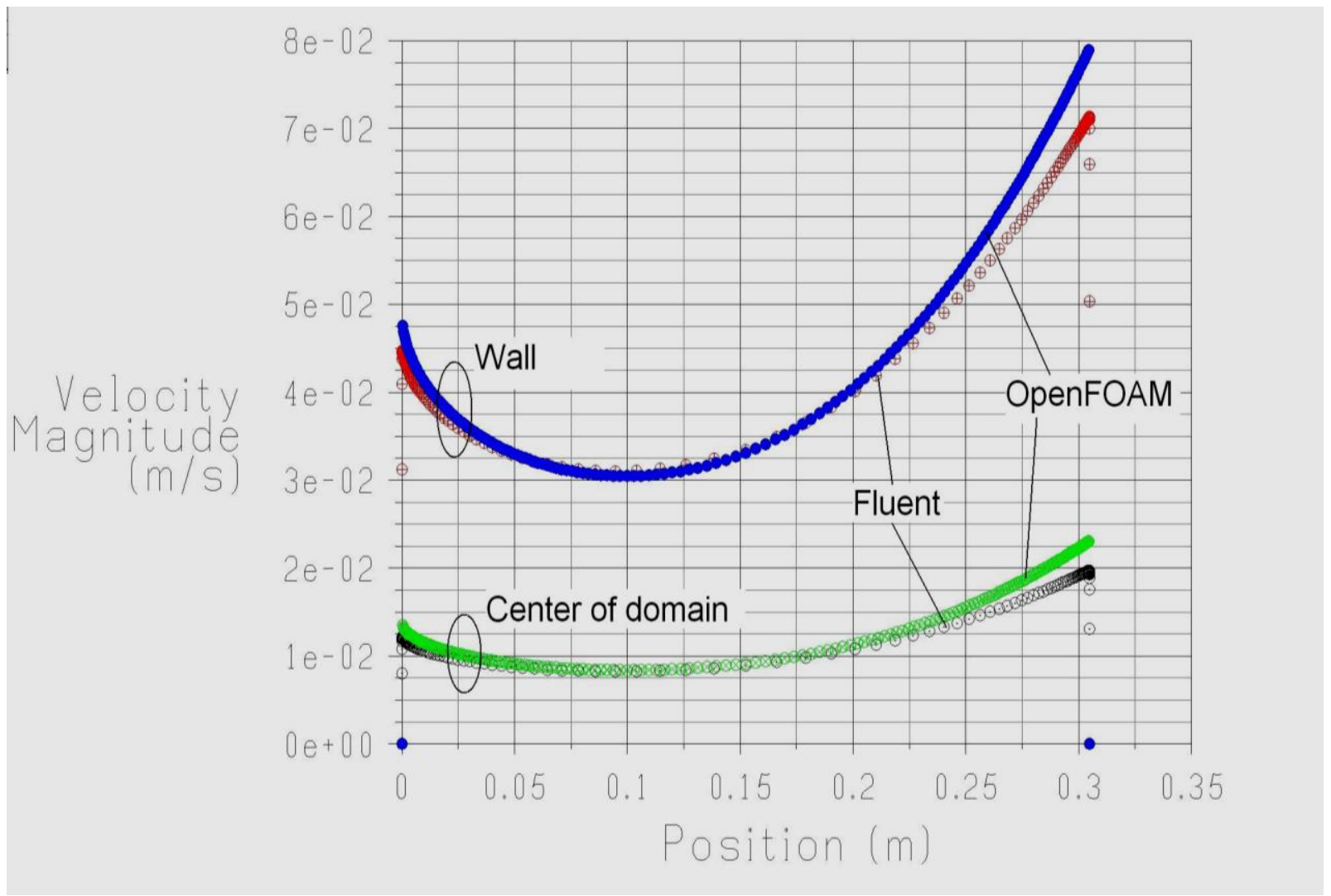

Figure 10: Wall velocity profiles along z-direction.

From above results, they can be seen that there is a good agreement between OpenFOAM simulation and Fluent. Velocity in outlet channel increases dramatically. While the velocity in inlet channel increases at entrance and obtains maximum at position of $50 \mathrm{~mm}$. This is due to hydrodynamic entry length. Then the velocity reduces until zero, as expected. Along length of the filter, wall velocity decreases up to position of $100 \mathrm{~mm}$ and then the wall velocity increases sharply. This can be explained by pressure difference of inlet and outlet channels which drives the wall velocity.

\section{1-DIMENSION DPF MODELING}

This study is to form a compact solver, 1D solver, which can be get results quickly with moderate accuracy according to a few input parameters. Figure 11 shows notation of the physical model. The pressure difference between inlet channel and outlet channel along the filter can be expressed as follows.

$$
\Delta \mathrm{p}=\mathrm{p}_{1}-\mathrm{p}_{2}=\mathrm{f}(\mathrm{U}, \mathrm{x}, \mathrm{w}, \mathrm{c}, \mathrm{L}, \rho, \mu, \alpha, \beta)
$$

where

$\begin{array}{ll}\mathrm{U} & \text { inlet velocity, } \mathrm{m} / \mathrm{s} \\ \mathrm{x} & \text { axial position, } \mathrm{m} \\ \mathrm{w} & \text { wall thickness, } \mathrm{m} \\ \mathrm{c} & \text { cell width, } \mathrm{m} \\ \mathrm{L} & \text { channel length, } \mathrm{m} \\ \rho & \text { density of working gas, } \mathrm{kg} / \mathrm{m}^{3} \\ \mu & \text { dynamic viscosity of working gas, } \mathrm{kg} / \mathrm{m} . \mathrm{s} \\ \alpha & \text { permeability, } \mathrm{m}^{2} \\ \beta & \text { inertial parameter, } 1 / \mathrm{m}\end{array}$




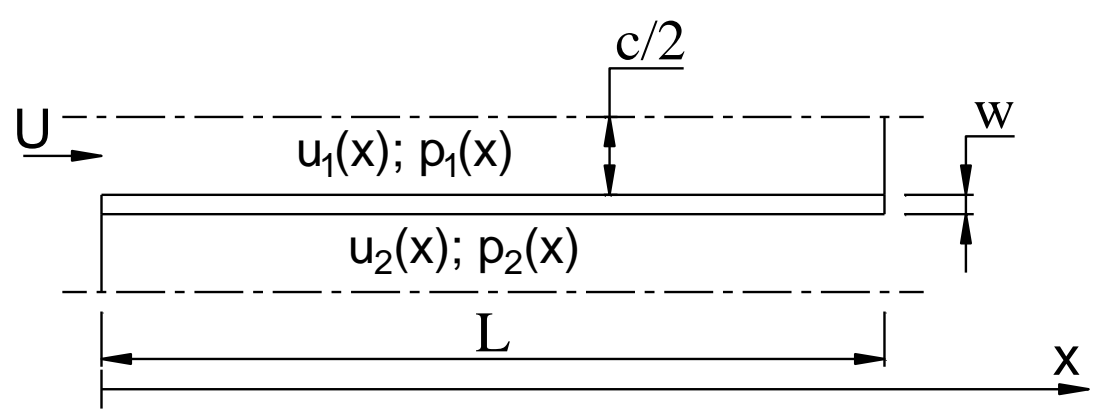

Figure 11: Schematic diagram of the wall-flow filter.

Assumptions were made for the model:

- Steady state

- Incompressible fluid

- 1 dimension

Mathematical model was formulated by 5 governing equations as

1. Pressure drop across the thin filter wall by using the modified Darcy's equation

where $u_{w}$ is wall flow velocity $(\mathrm{m} / \mathrm{s})$

$$
\mathrm{p}_{1}-\mathrm{p}_{2}=\frac{\mu}{\alpha} \mathrm{wu}_{\mathrm{w}}+\frac{1}{2} \beta \rho \mathrm{wu}_{\mathrm{w}}^{2}
$$

2. Momentum equations

where factor $\mathrm{F}$ equals to 28.454 .

$$
\begin{aligned}
\frac{\mathrm{du}_{1}^{2}}{\mathrm{dx}} & =-\frac{1}{\rho} \frac{\mathrm{dp_{1 }}}{\mathrm{dx}}-\mathrm{F} \frac{\mu}{\rho \mathrm{c}^{2}} \mathrm{u}_{1} \\
\frac{\mathrm{du}_{2}^{2}}{\mathrm{dx}} & =-\frac{1}{\rho} \frac{\mathrm{dp}_{2}}{\mathrm{dx}}-\mathrm{F} \frac{\mu}{\rho \mathrm{c}^{2}} \mathrm{u}_{2}
\end{aligned}
$$

3. Continuity equations

Rearrange the above equations as

$$
\begin{aligned}
& \frac{\mathrm{du}_{1}}{\mathrm{dx}}=-\frac{4}{\mathrm{c}} \mathrm{u}_{\mathrm{w}} \\
& \frac{\mathrm{du} \mathrm{u}_{2}}{\mathrm{dx}}=\frac{4}{\mathrm{c}} \mathrm{u}_{\mathrm{w}}
\end{aligned}
$$

Eq. 4 plus Eq.5 yields

$$
\begin{aligned}
& \frac{\mathrm{du}_{1}}{\mathrm{dx}}+\frac{\mathrm{du}_{2}}{\mathrm{dx}}=0 \\
& \Rightarrow \mathrm{d}\left(\mathrm{u}_{1}+\mathrm{u}_{2}\right)=0
\end{aligned}
$$

Apply B.C.: $\quad \mathrm{x}=0 \Rightarrow \mathrm{u}_{1}=\mathrm{U} ; \mathrm{u}_{2}=0$.

Therefore,

Eq. 3 minus Eq. 2 yields

$$
\mathrm{u}_{1}+\mathrm{u}_{2}=\mathrm{U}
$$

$$
\frac{\mathrm{d}\left(\mathrm{u}_{2}^{2}-\mathrm{u}_{1}^{2}\right)}{\mathrm{dx}}=\frac{1}{\rho} \frac{\mathrm{d}\left(\mathrm{p}_{1}-\mathrm{p}_{2}\right)}{\mathrm{dx}}-\frac{\mathrm{F} \mu}{\rho \mathrm{c}^{2}}\left(\mathrm{u}_{2}-\mathrm{u}_{1}\right)
$$

Substituting Eq. 1 and Eq. 6 into the above equation

$$
\frac{d\left(u_{2}^{2}-U^{2}+2 u_{2} U-u_{2}^{2}\right)}{d x}=\frac{1}{\rho} \frac{d\left(\frac{\mu}{\alpha} w u_{w}+\frac{1}{2} \beta \rho w u_{w}^{2}\right)}{d x}-\frac{F \mu}{\rho c^{2}}\left(u_{2}-U+u_{2}\right)
$$




$$
\begin{aligned}
& \underset{\text { Substituting } \mathrm{u}_{\mathrm{w}} \text { from Eq.5 }}{\longrightarrow} \frac{\mathrm{d}\left(2 \mathrm{u}_{2} \mathrm{U}-\mathrm{U}^{2}\right)}{\mathrm{dx}}=\frac{1}{\rho} \frac{\mathrm{d}\left[\frac{\mu}{\alpha} \mathrm{w} \frac{\mathrm{c}}{4} \frac{\mathrm{du}}{\mathrm{dx}}+\frac{1}{2} \beta \rho \mathrm{w} \frac{\mathrm{c}^{2}}{16}\left(\frac{\mathrm{du}}{\mathrm{dx}}\right)^{2}\right]}{\mathrm{dx}}-\frac{F \mu}{\rho c^{2}}\left(-U+2 \mathrm{u}_{2}\right) \\
& \Leftrightarrow 2 \mathrm{U} \frac{\mathrm{du}_{2}}{\mathrm{dx}}=\frac{1}{\rho}\left[\frac{\mu}{\alpha} \mathrm{w} \frac{\mathrm{c}}{4} \frac{\mathrm{d}^{2} \mathrm{u}_{2}}{\mathrm{dx}^{2}}+\frac{1}{2} \beta \rho \mathrm{w} \frac{\mathrm{c}^{2}}{16} \frac{\mathrm{d}}{\mathrm{dx}}\left(\frac{\mathrm{du}_{2}}{\mathrm{dx}^{2}}\right)^{2}\right]-\frac{\mathrm{F} \mu}{\rho \mathrm{c}^{2}}\left(-\mathrm{U}+2 \mathrm{u}_{2}\right) \\
& \Leftrightarrow \frac{\mathrm{d}^{2} \mathrm{u}_{2}}{\mathrm{dx}^{2}}-\frac{8 \rho \alpha \mathrm{U}}{\mu \mathrm{wc}} \frac{\mathrm{du}_{2}}{\mathrm{dx}}-\frac{8 \mathrm{~F} \alpha}{\mathrm{wc}^{3}} \mathrm{u}_{2}+\frac{4 \alpha \mathrm{F}}{\mathrm{wc}^{3}} \mathrm{U}+\frac{1}{2} \frac{\beta \mathrm{c} \rho \alpha}{2 \mu} \frac{\mathrm{du}_{2}}{\mathrm{dx}} \frac{\mathrm{d}^{2} \mathrm{u}_{2}}{\mathrm{dx}^{2}}=0 \\
& \text { Let } \quad \mathrm{A}_{1}=\frac{4 \rho \alpha \mathrm{U}}{\mu \mathrm{wc}}=\frac{4 \alpha}{\mathrm{wc}^{2}} \operatorname{Re} \quad \text { where } \mathrm{Re}=\frac{\rho \mathrm{Uc}}{\mu} \\
& \mathrm{A}_{2}=\frac{4 \alpha \mathrm{F}}{\mathrm{wc}^{3}} \\
& \lambda=\frac{\beta c \rho \alpha}{4 \mu}=\frac{\alpha \beta}{4 \mathrm{U}} \operatorname{Re}
\end{aligned}
$$

Then, the above equation becomes

$$
\frac{d^{2} u_{2}}{d x^{2}}-2 A_{1} \frac{d u_{2}}{d x}-2 A_{2} u_{2}+A_{2} U+\lambda \frac{d u_{2}}{d x} \frac{d^{2} u_{2}}{d x^{2}}=0
$$

B.C.: $\quad \mathrm{u}_{2}(0)=0$

$$
\mathrm{u}_{2}(\mathrm{~L})=\mathrm{U}
$$

To facilitate usage of the above formulation, the model was programmed using Microsoft Excel as a macro. The boundary value problem (Eq. 7) is solved by using shooting method. A lot of comparisons were performed to validate the $1 \mathrm{D}$ model. Figure 12 showed a typical comparison about pressure difference between inlet channel and outlet channel along the DPF. As can be seen, there is a good agreement between $3 \mathrm{D}$ and $1 \mathrm{D}$ approaches.

$\begin{array}{rll}\text { Inputs: } & & \\ \mathrm{m}=3 \mathrm{e}-5 & \mathrm{~kg} / \mathrm{s} \\ (\mathrm{U}=24.8 & \mathrm{m} / \mathrm{s}) \\ \mathrm{w}=0.432 \mathrm{e}-3 & \mathrm{~m} \\ \mathrm{c}=2.11 \mathrm{e}-3 & \mathrm{~m} \\ \mathrm{~L}=304.8 \mathrm{e}-3 & \mathrm{~m} \\ \alpha=2 \mathrm{e}-13 & \mathrm{~m}^{2} \\ \beta=0 & 1 / \mathrm{m}\end{array}$

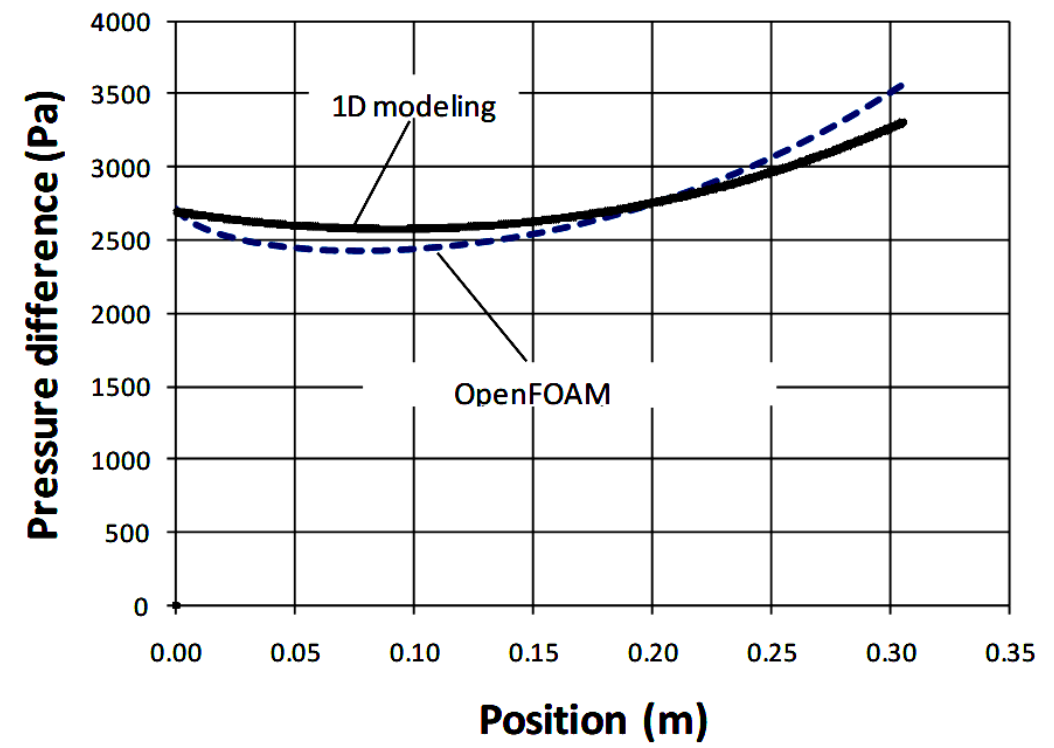

Figure 12: Test case for the 1D solver. 


\section{CONCLUSIONS}

3D simulation of flow and pressure in DPF was performed in this paper by using the open source CFD toolbox OpenFOAM. The results of simulation were compared with those of the commercial software Fluent. They show good agreement between two tools. To overcome disadvantage of both tools is that they take long time from pre-processing to post-processing for the problem. So, a compact 1D solver was developed. The model is based on Darcy equation and empirical coefficients. The 1D model was solved by using shooting method. Deviation between two approaches was negligible.

\section{REFERENCES}

[1] Athanasios, G.K., et al., Optimized Filter Design and Selection Criteria for Continuously Regenerating Diesel Particulate Traps. SAE 1999-01-0468.

[2] Gerd, G. and M. Patrick, Prediction of Pressure Drop in Diesel Particulate Filters Considering Ash Deposit and Partial Regenerations. SAE 2004-01-0158.

[3] Schejbal, M., et al., Modelling of diesel filters for particulates removal. Chemical Engineering Journal, 2009. 154(1-3): p. 219-230.

[4] Fluent 5 User's Guide

[5] Soldati, A., M. Campolo, and F. Sbrizzai, Modeling nano-particle deposition in diesel engine filters. Chemical Engineering Science, 2010. 65(24): p. 6443-6451.

[6] Liu, Y., et al., Nanoparticle motion trajectories and deposition in an inlet channel of wall-flow diesel particulate filter. Journal of Aerosol Science, 2009. 40(4): p. 307-323.

[7] Sbrizzai, F., P. Faraldi, and A. Soldati, Appraisal of three-dimensional numerical simulation for sub-micron particle deposition in a micro-porous ceramic filter. Chemical Engineering Science, 2005. 60(23): p. 65516563.

[8] www.ansys.com

[9] www.openfoam.com

\section{MÔ PHỎNG BA CHIỀU VÀ MỘT CHIỀU BỘ LỌC KHÓI ĐỘNG CƠ DIESEL}

Tóm tắt. DPF là một thiết bị quan trọng trong hệ thống lọc khói động cơ Diesel. Trong bài báo này chúng tôi mô phỏng phân bố vận tốc và áp suất trong DPF để xác định đặc tính động học và thủy lực, mhằm tạo cơ sở thiết kế và chọn lựa các kênh trong DPF. Mô phỏng số được thực hiện sử dụng phần mềm thương mại ANSYS Fluent và phần mềm mã nguồn mở OpenFOAM. Kết quả cho thấy sai lệch giữa hai phần mềm là không đáng kể. Một mô hình toán $1 \mathrm{D}$ gọn nhẹ được phát triển dựa trên phương trình Darcy, phương trình động lượng và phương trình liên tục. Mô hình toán được giải bằng phương pháp bắn cho bài toán giá trị biên. Kết quả mô phỏng từ chương trình $1 \mathrm{D}$ và $3 \mathrm{D}$ là rất trùng khớp nhau.

Từ khóa. Lọc khói động cơ Diesel, mô phỏng số, mô hình xốp, OpenFOAM, Fluent.

Ngày nhận bài: 27/02/2018

Ngày chấp nhận đăng: 12/12/2018 